\title{
ACTIVIDADES DA S.P.Q.
}

19. ENCONTRO INTERNACIONAL SOBRE EDUCAÇAOO EM QUIMMICA

ORGANIZADO POR: SOCIEDADE PORTUGUESA DE QUIMICA

EM COLABORAÇAOO COM: GRUPO DE TRABALHO SOBRE EDUCAÇAO DA FEDERAÇAO EUROPEIA DE SOCIEDADES QUIM.

LOCAL: LISBOA, LABORATORIO NACIONAL DE ENGENHARIA CIVIL

DATAS: $18-19-20$ de OUTUBRO de 1978

(Para informações complementares veja Boletim no 4 ou escreva para o Secretariado da S.P.Q.; tel. 572616) 


\section{ENCONTRO NACIONAL DE QUIMICA}

Organizado pela Sociedade Portuguesa de 2uimica, realizar-se-ā, nos dias 3,4 e 5 de Janeiro de 1979, o segundo Encontro Nacional de Quimica.

Para informações complementares, contactar para:

Sociedade Portuguesa de 2uimica

Delegação do Porto

Departamento de Quimica

Faculdade de Ciências

PORTO

Portugal 